\title{
J.H. von Thünens Eintreten für die Gründung eines „Mecklenburgischen Ritterschaftlichen Credit-Vereins“ - eine gemeinwirtschaftliche Unternehmensinitiative
}

Frei-gemeinwirtschaftliche Kreditgenossenschaften; gemeinwirtschaftliche Unternehmen; öffentlich-rechtliche Realkredit-Institute

In der auf Thünen bezogenen Forschung wurde bisher zu wenig beachtet, dass er neben seinem klassischen volkswirtschaftlichen und zugleich agrarwissenschaftlichbetriebswirtschaftlichen Hauptwerk „,Der isolierte Staat in Beziehung auf Landwirtschaft und Nationalökonomie "l zahlreiche größere und kleinere Beiträge in seinem ständischen Mecklenburger Landesorgan, den „Neuen Annalen der Mecklenburgischen Landwirtschaftsgesellschaft", über betriebswirtschaftliche, technologisch-technische und finanzielle Fragen veröffentlicht hat. Einen besonderen Rang unter diesen Abhandlungen nehmen zwei Beiträge ein: „Ansichten über die Gründung eines landwirtschaftlichen Instituts in Mecklenburg ", ${ }^{2}$ in dem für eine theoretisch und praktisch orientierte liberale Agrarlehre auf der Basis der klassischen Nationalökonomie plädiert wird, und bereits vorher die Arbeit „Über die Einführung eines Kreditsystems in Mecklenburg und über die Bestimmung des Pfandwertes der Mecklenburgischen Landgüter “. 3

I. Bei dem deutschen Klassiker der Nationalökonomie und landwirtschaftlichen Betriebslehre gibt es noch immer Wichtiges zu entdecken oder zumindest neu zu bewerten

Johann Heinrich von Thünen wird in der Gegenwart zu recht v.a. als ,liberal“ eingestellter landwirtschaftlicher Unternehmer und als herausragender nationalökonomischer Spätklassiker, aber auch als ein vom frühsozialistischen Schriftum zum eigenen Nachdenken und Handeln anregender ,sozialer“ Entrepreneur gewürdigt. Er bezeichnete bekanntlich Albrecht Thaer und Adam Smith als seine „Lehrer“ in der Landwirtschaft und der Natio-

\footnotetext{
Siehe die von H. Schumacher herausgegebene Gesamtausgabe der III Teile des Werks: Thünen (1875).

Vgl. Thünen (1831), S. 282-322.

Vgl. Thünen (1817), S. 403-545.
} 
nalökonomie, wurde aber auch erheblich durch Immanuel Kants „Kritik der reinen Vernunft" beeinflusst. ${ }^{4}$

Obwohl unter den Göttinger Hochschullehrern während seines kurzen Göttinger Universitätsstudiums und von den später von seinem Landgut Tellow aus an der Universität Rostock und mehreren Regierungsstellen kontaktierten Fachleuten und Politikern die überwiegend kameralistisch oder doch britisch-merkantilistisch geprägten Personen noch zweifellos dominierten - und er selbst sogar einmal eine Chance zu haben schien, selbst als Nachfolger eines Kameralisten an die Universität Rostock berufen zu werden, ${ }^{5}$ ist seine Lebensleistung tatsächlich bereits unzweifelhaft primär unter marktwirtschaftlichen Aspekten ohne wesentlichen Staatseinfluss zu sehen. ${ }^{6}$

Gleichwohl sollte nicht übersehen werden, dass sich sowohl sein fortschrittliches inventives Denken als auch sein lebenslang praktiziertes innovatives Handeln öffentlichen bzw. gemeinwirtschaftlichen Fragen keineswegs völlig verschlossen hat. Er verstand diese Probleme durchaus als solche der Staatswirtschaft oder aber als solche des kooperativen Handelns. Unter dem Einfluss des kantischen Strebens nach Klärung und Verwirklichung von mehr Gerechtigkeit und Freiheit in der Wirtschaft und Gesellschaft und in Anbetracht der von ihm neben zahlreichen liberalen marktwirtschaftlichen Abhandlungen begrenzt auch verarbeiteten staatswirtschaftlichen Schrifttums, v.a. solchem im Sinne des rationalen Naturrechts, ${ }^{7}$ wäre anderes auch kaum zu erwarten.

Der Gesamtansatz seines Wollens, Forschens und praktischen Verhaltens ging damit im Grunde von einer gemischten Wirtschaftsordnung aus, wie sie beispielsweise Peter Eichhorn charakterisiert hat. ${ }^{8}$ Thünens Bestreben war sogar bereits auf Elemente einer Dritten Ordnung von Staat und Gesellschaft unter Einbeziehung des Sozialen sowie beibehaltener oder neugegründeter staatlicher und genossenschaftlicher Unternehmen gerichtet, und nicht auf eine rein marktwirtschaftlich allein durch erwerbswirtschaftlich handelnde private Unternehmen geprägte Ordnung. ${ }^{9}$

Dies alles lässt sich besonders an seiner Lohntheorie und deren praktischer Umsetzung demonstrieren, welche entschieden einem produktivitätsorientierten, durchaus auch genossenschaftsnahen Ansatz folgte. Am Ende seines Lebens bejahte Thünen sogar bereits eine bürgerschaftlich initiierte Mecklenburger Region zwischen Teterow und Güstrow als Zentrum künftigen weiteren wirtschaftlichen und sozialen Fortschritts. ${ }^{10}$

Insbesondere Vertreter der institutionalistisch argumentierenden älteren Historischen Schule in Deutschland haben bezogen auf die verkehrs- und handelspolitischen Anschauungen Thünens sein kombiniert gemeinwirtschaftlich-privatwirtschaftliches Denken und Handeln früh richtig gesehen. So hob Wilhelm Roscher seine Bejahung des „Staatsbaus“ der Eisenbahnen neben privatem Straßen- und Wegebau hervor, „damit durch gemein-

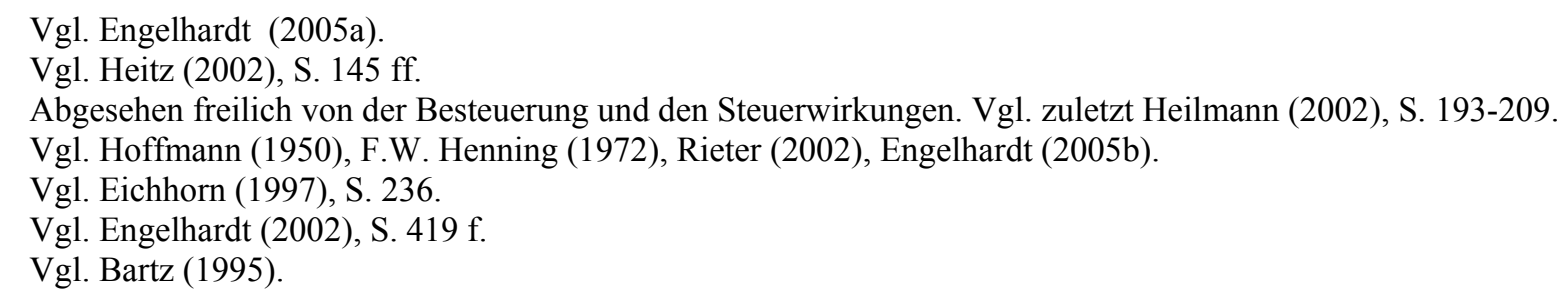


nützigen Verzicht auf eigentlichen Gewinn die Frachtpreise der Güter bedeutend erniedrigt werden könnten“. 11

\section{Argumente Thünens für die Einführung eines Kreditinstituts in Analogie zu den preußischen Landschaften, aber mit abweichenden spezifischen Merkmalen}

Bei einer Würdigung des kreditwirtschaftlichen Thünenschen Ansatzes ist davon auszugehen, dass zur Linderung der Kreditnot der durch den Siebenjährigen Krieg sehr hart mitgenommenen schlesischen Rittergutsbesitzer zuerst Friedrich der Große ab 1770 sogenannte „Landschaften“ gegründet hat. Dies erfolgte über Schlesien hinaus auf der Basis bereitgestellter Fonds bspw. auch in Pommern. Die Gründungen stellten vom Landesherrn verordnete Zusammenschlüsse, d.h. staatliche Unternehmen in öffentlichrechtlicher Form dar, zwecks gemeinsamer - quasi zwangsgenossenschaftlicher - Haftungsübernahme der Gläubiger für auszugebende börsengängige „Pfandbriefe“ für langfristige Investitionskredite.

Außerhalb Preußens und eben auch in den beiden Teilen Mecklenburgs kam es später bald zu Abwandlungen der ursprünglich angewandten landschaftlichen Organisationsform. Die anfängliche Exklusivität der Landschaften, die nur zur Befriedigung der Kreditbedürfnisse des meist adligen Großgrundbesitzes gedacht waren und sich schnell zu machtvollen Standes-Organisationen entwickelten, wurde v.a. nach 1848 zugunsten des bäuerlichen Grundbesitzes aufgelockert, ${ }^{12}$ in Mecklenburg dank Thünen bei freiwilliger Mitgliedschaft sogar bereits 1818.

In jenem Jahr kam es nicht nur zur Gründung, sondern auch bereits zur landesherrlichen Bestätigung eines ritterschaftlichen Kredit-Vereins nebst Statuten und Anlagen für alle drei Kreise der beiden Großherzogtümer Mecklenburgs. Beitrittsberechtigt waren auch bürgerliche Besitzer von Gütern, die zum ritterschaftlichen Kataster gehörten und in einer der beiden ritterschaftlichen „Brand-Societäten“ versichert waren. Ausgeschlossen blieben hingegen Güter, die sich im Besitz der Landesherrschaft befanden, was bei 40\% des Landes der Fall war.

In der umfangreichen Abhandlung Thünens wird im Ersten Abschnitt zunächst die Notwendigkeit eines Kreditsystems erläutert. Sodann folgen im Zweiten Abschnitt sehr detaillierte, durch empirisches Zahlenmaterial gestützte Überlegungen über die geeigneteste Methode zur Bestimmung des Pfandwerts der Landgüter. Diese Darlegungen nehmen nicht weniger als zwei Drittel der Abhandlung in Anspruch, wobei auch ausgiebig auf die im Mecklenburgischen Landesvergleich staatlicherseits vorgeschriebenen „BonitierungsReglements“ bezug genommen wird.

11 Vgl. Roscher (1874), S. 897 f. bezogen auf Thünen (1875), II,2, S. 83 ff. und 244 ff. Der Klassiker bejaht Bahnen in den Händen des Staates, weil so ,die Güterfracht auf die Hälfte herabgesetzt und doch die Zinsen des Anlagekapitals gedeckt werden“ könnten; vgl. Thünen (1875), II,2, S. 83.

12 Vgl. Meinhold (1956), S.61. 
Für Thünen war „die Wohltat eines Kreditvereins... schon durch das Pommersche System bestätigt, das indessen für Mecklenburg nur unter Beschränkungen zum Muster dienen kann“, weil hier teilweise andere Umstände zu berücksichtigen seien. Er fragt: „Wie könnte die Befolgung des im Mecklenburgischen Landesvergleich vorgeschriebenen Bonitierungs-Reglements eine möglichst genaue Annäherung zum richtigen Resultat geben?“. Es ging Thünen also primär um einen genau spezifizierten Anschlag zur richtigen Schätzung des Pfandwerts. Für ihn war es die entscheidende Frage, ob der Pfandwert so genau bestimmt werden könne,, ,dass dadurch der große Zweck des Kreditvereins, das unbedingte Vertrauen Aller zu gewinnen, erreicht werde“. ${ }^{13}$

Um dem potentiellen Kreditgeber Sicherheit zu bieten, schlug der Autor vor:

„1. Die Einführung von unablöslichen Rentenzahlungen statt der bisherigen Kapitalforderungen, wenigstens für die zur ersten Hypothek stehenden Kapitalien.

2. Die gerichtliche Eintragung der Renten.

3. Eine glaubwürdige gerichtliche Taxe des der Rente zur Hypothek dienenden Guts.

4. Verminderung der Unkosten beim Verkauf eines Guts“. ${ }^{14}$

Durch den ersten Vorschlag wurde das Versprechen des Gutsbesitzers, Zinsen zu zahlen, glaubhaft gemacht und damit die Ungewissheit für den Kreditgeber erheblich reduziert. Nur dann aber, wenn die Rente gerichtlich eingetragen und von einer ebenfalls eingetragenen Hypothek gedeckt wurde - die Vorschläge 2 und 3 -, konnte sie allgemein als sicher und auf dem Kapitalmarkt als verkäuflich gelten. Dabei sollte der Wert der Hypothek so bemessen sein, dass das Gut einen Ertrag abwirft, der auch im schlechtestmöglichen Zustand die Rente übersteigt. Zur Änderung der ihm als weithin ablehnend bekannten Meinung des Publikums schlug Thünen vor, dass ,alle Gutsbesitzer sich gegenseitig für die auf ihren Gütern haftenden Renten verbürgen, und somit das ganze Land eine gemeinsame Hypothek für die Rente wird““. ${ }^{15}$

Was den vierten Punkt, die Verminderung der laufenden Kosten der Administration betrifft, weicht Thünens Vorschlag allerdings erheblich von der Praxis der Preußischen Landschaften als Staatsunternehmen ab. Ein Kreditverein lohnt sich seiner Ansicht nach nur dann, wenn die Kosten seiner Verwaltung nicht seine Vorteile übersteigen. Den kritischen Wert, bei dem diese Kosten zu hoch werden, schätzte er auf 0,5\% des geliehenen Kapitals. Während z.B. die Pommersche Landschaft etwas weniger als $1 \%$ für die Kosten der Administration benötigte. Thünen empfahl deshalb, die Verwaltung privatwirtschaftlich zu betreiben und sogar auf einen öffentlichen Fonds zu verzichten. „Die Erfahrung aller Zeiten hat es bewiesen, dass jedes einer Kommune gehörige Vermögen nicht so gut verwaltet wird, und nicht so vielen Ertrag bringt, als wenn dies Vermögen in den Händen der Privatleute ist".

„Es wäre also zu wünschen, dass der Kreditverein keines bedeutenden, der ganzen Gesellschaft gemeinschaftlich gehörenden Fonds - der nur durch Verminderung des Privatvermögens gebildet werden kann - bedürfe“. Thünen plädiert nur für ein „kleines bares

13 Vgl. Thünen (1817), S. 406 ff. und 446 ff., hier 450 und 465 ff.

14 Vgl. Thünen (1817), S. $417 \mathrm{f}$.

15 Vgl. Thünen (1817), S. 428. 
Kapital" und zwecks Vereinfachung der Administration auch dafür, dass der Verein keine regelmäßigen Geschäfte tätigen solle und an Personal spare. Die Zinsen sollten direkt vom Schuldner an den Gläubiger - unter Umgehung des Vereins - gezahlt werden, und die Pfandbriefe sollten für Gläubiger und Schuldner unkündbar sein. „Wenn die direkte Zinszahlung beibehalten wird, so hat das Direktorium eigentlich gar keine regelmäßigen Geschäfte. Es müsste nur immer bereit sein, um dann, wenn eine Stockung in dem Gange der Maschine entstände, tätig einzugreifen und die Ordnung wieder herzustellen" ${ }^{16}$

Das zentrale Element des Thünen`schen Vorschlags war aber zweifellos die valide Wertermittlung der als Sicherheit für die Pfandbriefe beliehenen Rittergüter. Unter dem Pfandwert eines Gutes verstand der Autor dabei den Wert, „der auch bei den ungünstigsten Konjunkturen noch übrig bleibt, und der als unverlierbar mit Pfandbriefen belastet werden kann“. ${ }^{17}$ „Der eigentliche Charme des Vorschlags liegt“ - wie Wolfram Rauch und Dirk Schiereck bestätigt haben - ,in der Kombination einer fundierten Bewertungsformel unter gleichzeitiger Zugrundelegung bereits vorhandener allgemein zugänglicher Qualitätsklassifikationen der landwirtschaftlichen Flächen“.18

\section{Statt eines gemeinwirtschaftlichen Staatsunternehmen, das es der Rechtsform nach wurde und blieb, ging es dem Klassiker bereits um ein frei-gemeinwirtschaftliches, genossenschaftsähnliches Institut}

Thünen stellte sich durch seine Arbeit von 1817 nach dem Urteil des landwirtschaftlichen Betriebslehrers und Agrarpolitikers Asmus Petersen, des wohl bedeutendsten agrarwissenschaftlichen Thünen-Forschers des vorigen Jahrhunderts, mit einem Schlage unter die Meister der landwirtschaftlichen Taxationslehre. Sie machte ihn zum Begründer der landwirtschaftlichen Rentenfondstheorie, die später auch von Karl Rodbertus-Jagetzow vertreten wurde und oft nur noch unter dem Namen „Rodbertus`sche Rentenfondstheorie" bekannt ist. ${ }^{19}$

Doris Neuberger sieht Thünen zu recht nicht nur als Förderer der Finanzintermediation, indem er auf seinem Landgut die beschäftigten Landarbeiter zur Spartätigkeit anregte und das Ersparte verwaltete. Sie betrachtet ihn auch als eigentlichen Begründer einer Theorie über die Einführung und Durchführbarkeit eines Kreditvereins. Der Verein war den preuBischen Landschaften ähnlich, hatte im Unterschied zu diesen allerdings keinen Mitgliederzwang und kam ohne Zwangsverwaltungen aus. Die Besicherung und Diversifikation der Kredite wurden als wichtige Merkmale eines Kreditinstituts beschrieben. „Derartige Erkenntnisse werden erst seit einigen Jahren innerhalb der Theorie unvollkommener Finanzmärkte mathematisch formalisiert “ ${ }^{20}$

16 Vgl. Thünen (1817), S. 430 ff. und 436.

17 Vgl. Thünen (1817), S. 439.

18 Vgl. Rauch/Schiereck (2002), S. $181 \mathrm{ff}$.

19 Vgl. Petersen (1944), S.10.

20 Vgl. Neuberger (1997), S. 101 f. 
Als landwirtschaftlicher Praktiker gab Thünen nach den umfassenden Darlegungen von Wolfram Rauch und Dirk Schiereck auch zahlreiche Anregungen und Anweisungen zu den Satzungsbestimmungen, wie die Mecklenburger Gründung als Körperschaft des öffentlichen Rechts unter der Oberaufsicht des Landesherrn konkret installiert werden sollte und wurde. Der Verein selbst umfasste im Jahr 182578 Güter, 1848116 Güter und entwickelte sich bis 1945, als er in der Sowjetischen Besatzungszone beseitigt wurde, zum zweitgrößten Kredit-Institut dieser Art in ganz Deutschland. ${ }^{21}$

Laut Neuberger wurde in der frühen Veröffentlichung Thünens bereits der spezifische methodologische Ansatz sichtbar, der für das wissenschaftliche Hauptwerk des Klassikers als charakteristisch gilt. „Er stellt präzise Beobachtungen an und sammelt alle verfügbaren Daten, um damit Berechnungen anzustellen. Dies geschieht nicht zufallsmäßig, sondern im Hinblick auf die „Potenzen“, die für den Untersuchungszweck wichtig sind und durch vorausgehende Überlegungen isoliert wurden“. ${ }^{22}$ Rauch/Schiereck haben in diesem Zusammenhang zu recht von einer ,intersubjektiv überprüfbaren empirischen Fundierung" des Aufsatzes durch Thünens berühmte langjährige Buchführung für sein Landgut Tellow gesprochen. ${ }^{23}$

Von den hervorgehobenen Merkmalen der Abhandlung Thünens her ist es realistisch, von einer von Anfang an gemeinwirtschaftlichen Struktur des neuen Instituts zu sprechen. In ihr haben stets eindeutig Sachziele im Sinne der grundsätzlichen Klärungen Peter Eichhorns und anderer Theoretiker der Gemeinwirtschaftstheorie im Vordergrund der Geschäftspolitik gestanden. ${ }^{24}$ Die auf Freiwilligkeit beruhende mitgliedschaftliche Ausrichtung des Kreditinstituts entsprach dem finanzorientierten frei-gemeinwirtschaftlichen Fördergedanken der Genossenschaften. Dies etwa im Sinne Friedrich Wilhelm Raiffeisens, der bekanntlich im Unterschied zu anderen Genossenschaftspionieren deutlich auch die gemeinwirtschaftliche Orientierung seiner Genossenschaften betont hat. ${ }^{25}$

Als Selbsthilfeeinrichtungen sollten die ritterschaftlichen Kreditinsitute nach Dirk Schierecks begründeter Ansicht zunächst v.a. dazu dienen, den Finanzbedarf der landund forstwirtschaftlichen Betriebe im Eigentum der Ritterschaftsmitglieder zu decken. Bereits insofern entsprach der gegründete Kreditverein der Rolle eines Unternehmens gemeinwirtschaftlicher Art. „Durch die Ausweitung des Kundenkreises über die Einbeziehung landwirtschaftlicher Unternehmer, die keine Ritterschaftsmitglieder sind, wurde das Geschäftsziel dahingehend erweitert, dass nunmehr allgemein eine günstige Realkreditvergabe zur Förderung des ländlichen Raumes verfolgt wird“ “.

21 Vgl. Rauch/Schiereck (2002), S. 183 ff. und 187 ff.

22 Vgl. Neuberger (1997), S. $101 \mathrm{f}$.

23 Vgl. Rauch/Schiereck (2002), S. 178.

24 Vgl. Eichhorn (1984), S. 244.

25 Vgl. Engelhardt (1983).

26 Vgl. Schiereck (1998), S. 367 f. 


\section{Literaturverzeichnis}

Bartz, Rolf-Peter (1995), Die Gegend zwischen Teterow und Güstrow - ein Zentrum des Fortschritts, in: Das Heimatjahrbuch für den neuen Landkreis Güstrow, S. 90-98

Eichhorn, Peter (1984), Betriebswirtschaftslehre und Gemeinwohl, in: Zeitschrift für Betriebswirtschaft, 54. Jg., S. 238-251

Eichhorn, Peter (1997), Öffentliche Betriebswirtschaftslehre, Baden-Baden

Engelhardt, Werner Wilhelm (1983), Gemeinwirtschaftliche Genossenschaften - ein möglicher Widmungstyp von Genossenschaften unter sechsen, in: Zeitschrift für öffentliche und gemeinwirtschaftliche Unternehmen, Bd. 6, S. 30-47

Engelhardt, Werner Wilhelm (2002), J.H. von Thünen als Vorläufer der Sozialen Marktwirtschaft, in: Zeitschrift für öffentliche und gemeinwirtschaftliche Unternehmen, Bd. 25, S. 406-420

Engelhardt, Werner Wilhelm (2005a), Wider das „Gesetz des Dschungels“": Johann Heinrich von Thünens Eintreten für moralisch fundierten Kapitalismus unter dem Einfluß von Adam Smith und Immanuel Kant, in: Familienpolitik und soziale Sicherung. Festschrift für Heinz Lampert, hrsg. von Jörg Althammer, Berlin, S. 453-479

Engelhardt, Werner Wilhelm (2005b), Zu den moralischen und wissenschaftstheoretischen Grundlagen der Wirtschafts- und Sozialpolitik in offenen Gesellschaften und demokratischen Staaten. J.H. von Thünens Beitrag unter dem Einfluß von A. Smith und I. Kant, in: Wirtschaftspolitik in offenen Demokratien. Festschrift für Uwe Jens, hrsg. von Lothar F. Neumann und Hajo Romahn, Marburg, S. 13-37

Heilmann, Martin (2002), Thünens Ideen zur modelltheoretischen Analyse der Steuerwirkungen, in: Berichte über Landwirtschaft, 215. Sonderheft, S. 193-209

Heitz, Gerhard (2002), Johann Heinrich von Thünen - ein vergeblicher Versuch seiner Berufung, in: Berichte über Landwirtschaft, 215. Sonderheft, S. 145-151

Henning, Friedrich-Wilhelm (1972), „Die große Stadt in verschiedenen Verhältnissen betrachtet“ (J.H.G. v. Justi, 1764), in: Zeitschrift für Agrargeschichte und Agrarsoziologie, 20. Jg., S. 186-197

Hoffmann, Friedrich (1950), J.H. v. Thünen im Blickfeld des deutschen Kameralismus, in: Weltwirtschaftliches Archiv, Bd. 65, S. 25-40

Meinhold, Wilhelm (1956), Agrarkredit, in: Handwörterbuch der Sozialwissenschaften, 1. Band, S. 59-66

Neuberger, Doris (1997), Johann Heinrich von Thünens Beiträge über Finanzintermediation, in: Bankhistorisches Archiv. Zeitschrift für Bankgeschichte, Bd. 24, S. 94-103

Petersen, Asmus (1944), Thünens Isolierter Staat, Berlin

Rauch, Wolfram und Schiereck, Dirk (2002), Thünens Vorschlag für einen Mecklenburgischen Ritterschaftlichen Credit-Verein - Bedeutung und Auswirkungen, in: Berichte über Landwirtschaft, 215. Sonderheft, S. $173-192$

Rieter, Heinz (2002), Johann Heinrich von Thünens nachgelassenes Manuskript über „Nationalökonomie“, in: Berichte über Landwirtschaft, 215. Sonderheft, S. 210-227

Roscher, Wilhelm (1874), Geschichte der National-Ökonomik in Deutschland, München

Schiereck, Dirk (1998), Ritterschaftliche Kreditinstitute in Deutschland, in: Zeitschrift für öffentliche und gemeinwirtschaftliche Unternehmen, Bd. 21, S. 360-369

Thünen, Johann Heinrich von (1817), Über die Einführung eines Kreditsystems in Mecklenburg und über die Bestimmung des Pfandwerts der Mecklenburgischen Landgüter, in: Neue Annalen der Mecklenburgischen Landwirtschafts-Gesellschaft, 4. Jg., S. 401-544

Thünen, Johann Heinrich von (1831), Ansichten über die Gründung eines landwirtschaftlichen Instituts in Mecklenburg, in: Neue Annalen der Mecklenburgischen Landwirtschafts-Gesellschaft, 17. Jg. S. 282-322

Thünen, Johann Heinrich von (1875), Der isolierte Staat in Beziehung auf Landwirtschaft und Nationalökonomie, I., II.1., II.2. und III. Teil, hrsg. von Hermann Schumacher, Berlin 